practices to prevent patient-to-patient transmission Infect Control Hosp Epidemiol 2003;24(Suppl 5):S1-52.

2. Siegel JD, Rhinehart E, Jackson M, et al, the Healthcare Infection Control Practices Advisory Committee. 2007 Guideline for Isolation Precautions. Preventing
Transmission of Infectious Agents in Healthcare Settings. http://www.cdc.gov/ncidod/dhqp/pdf/guidelines/ Isolation2007.pdf (accessed 18 Aug 2009).

3. Wainwright CE, France MW, O'Rourke P, et al.

Cough-generated aerosols of Pseudomonas aeruginosa and other Gram-negative bacteria from patients with cystic fibrosis. Thorax 2009;64:926-31.

4. Roy CJ, Milton DK. Airborne transmission of communicable infection - the elusive pathway. N Engl J Med 2004;350:1710-2

\title{
Medication adherence in COPD: what have we learned?
}

\section{MeiLan K Han}

As physicians, we take great interest in the adherence of our patients to prescribed medications. This is based on our belief that the benefits of treatment will be greater in patients who take their medication regularly and less among those who do not. C Everett Koop is quoted as having said: "Drugs don't work in patients who don't take them". The practice of faithfully complying with a medication regimen, however, may be just as important as the efficacy of the medication itself. In this month's issue of Thorax, Vestbo et al ${ }^{1}$ report that, among patients with chronic obstructive pulmonary disease (COPD) participating in the TORCH trial, ${ }^{2}$ adherers experienced significantly better survival and a lower risk of hospital admission due to exacerbations than non-adherers (see page 939). These effects, however, were independent of treatment group.

In fact, a growing body of literature suggests that adherence to drug therapy in clinical trials-both medication and placebo regimens-predicts better outcomes. Many of these data have been generated from cardiovascular trials. ${ }^{3}$ In 1980 a report from the Coronary Drug Project significantly challenged traditional scientific thought regarding the relationship between adherence and disease outcomes. $^{4}$ This randomised double-blind placebo controlled trial examined the effects of clofibrate on the survival of men who had experienced a myocardial infarction. ${ }^{5}$ Five-year mortality was similar for those treated with medication $(20 \%)$ and those treated with placebo (21\%). When analysed by adherence patterns, however, good adherers (defined as those taking at least $80 \%$ of the prescribed medication) had significantly lower 5-year mortality than poor adherers (15\% vs 25\%). Surprisingly, however, these findings were nearly identical in the

Correspondence to: $\mathrm{Dr} \mathrm{M} \mathrm{K}$ Han, University of Michigan, 1500 E Medical Center Drive, 3916 TC, Ann Arbor, Ml 48109-5360, USA; mrking@umich.edu placebo group (15\% vs $28 \%$ mortality). Initially, much discussion focused on the potential for bias in post hoc analyses. As more data have accumulated, however, the scientific community has begun to realise that, across disciplines and treatment assignments, adherent behaviour in clinical trials is associated with better outcomes. ${ }^{3}$

While it has been hypothesised that improved outcomes for adherers may be due to selection bias with good adherers having less severe disease, this is probably not the case. ${ }^{4}$ The improved outcome for adherers in the study by Vestbo et al remained after adjustment for disease severity as measured by forced expiratory volume in $1 \mathrm{~s}$ and dyspnoea measured by the MRC dyspnoea scale. ${ }^{1}$ Similar results have been reported in other trials. ${ }^{4}$ In fact, the Lung Health Study reported that better adherence was associated with more severe airways obstruction. ${ }^{6}$ Another hypothesis is that adherence to drug treatment may reflect a patient's biological, social and psychological makeup which may all influence outcomes. Adherence may be a surrogate marker for other healthy behaviours such as taking medications for comorbid conditions, eating more healthily or taking safety precautions such as wearing seatbelts or sunscreen. Alternatively, however, the adherence itself and the expectation of treatment effect may activate behaviours that are then partly responsible for improved outcomes. ${ }^{7}$ For instance, the act of adhering to a treatment regimen may instil a sense of well-being and reduce anxiety about a chronic disease or may lead to altered health habits such as exercise or smoking cessation.

Very few data exist regarding adherence to inhaled medication in COPD. It was previously reported that only about 37\% of patients with chronic lung disease are fully adherent with medical treatments in general. ${ }^{8}$ Long-term adherence with inhaled medications was reported from the Lung Health Study. ${ }^{6}$ Self-reported data suggested that nearly $70 \%$ of patients adhered to the medication regimen, dropping off slightly over the next 18 months. Adherence confirmed by canister weights, however, showed that only $48 \%$ of patients had good adherence at 1 year. Two other studies of nebulised treatments in COPD suggest that only about half of patients take their medications regularly. ${ }^{9}{ }^{10}$ In the current study, $80 \%$ of subjects demonstrated good adherence (defined as adherence to study medications of $>80 \%$ over the entire period the subject was in the study). While this is a good percentage within the context of a clinical trial, it probably does not reflect "real life" patient behaviour.

One would hope that the patients who need medication most might also be the group that is most adherent. It is therefore disappointing that, in the study by Vestbo et $a l,{ }^{1}$ no association was found between disease severity defined by GOLD stage and adherence. In fact, poor adherers actually had higher MRC dyspnoea scores. However, it should also be noted that adherence in this study was determined by the percentage of returned drug left unused. What this analysis does not take into account is the significantly greater number of patients who dropped out of the placebo arm compared with the combination therapy arm $(44.2 \%$ vs $34.1 \%$ ), which also could be interpreted as non-adherence in the broadest sense of the word. These data suggest that adherence in this more global sense is probably less than the reported $80 \%$. They also suggest that symptomatic improvement or drug efficacy may have had an impact on study participation even if the analysis of medication adherence among those who stayed in the trial did not differ between treatment and placebo arms.

In summary, this study indicates that adherence to inhaled therapy in COPD within the context of a clinical trial is good and associated with improved outcomes. Ultimately, however, if this is due to qualities intrinsic to the adherer, this actually leaves little room for the physician to modify outcomes other than to prescribe the treatment itself. If, however, the act of adhering actually activates other positive behaviours, then we as physicians should be even more aggressive about creating structured treatment 
regimens (simulating a clinical trial) for our patients. In a study evaluating metered dose inhaler compliance in COPD, use of an electronic medication monitor with feedback on the accuracy of medication use improved compliance. ${ }^{11}$ This type of methodology could be used to improve adherence in COPD outside a clinical trial. Clearly, further research is needed to better understand the factors driving the phenomenon of improved outcomes in adherers. While it is disappointing that neither treatment with active drug nor disease severity had an impact on adherence, study participation was better in the combination treatment arm of the TORCH study which suggests that symptomatic improvement may positively affect the likelihood of compliance of a patient with COPD in the more global sense. This would be good news for our patients as the current analysis also suggests that the association between adherence and mortality risk reduction with combination therapy was even greater when only those in the treatment arm were analysed.

Competing interests: None.

Provenance and peer review: Commissioned; not externally peer reviewed.

Thorax 2009;64:922-923. doi:10.1136/thx.2009.121806

\section{REFERENCES}

1. Vestbo J, Anderson JA, Calverley PMA, et al. Adherence to inhaled therapy, mortality and hospital admission in COPD. Thorax 2009;64:939-43.

2. Rabe KF, Hurd S, Anzueto A, et al. Global strategy for the diagnosis, management, and prevention of chronic obstructive pulmonary disease: GOLD executive summary. Am J Respir Crit Care Med 2007;176 532-55.

3. McDermott MM, Schmitt B, Wallner E. Impact of medication nonadherence on coronary heart disease outcomes. A critical review. Arch Intern Med 1997;157:1921-9.
4. Horwitz RI, Horwitz SM. Adherence to treatment and health outcomes. Arch Intern Med 1993;153:1863-8

5. Anon. Influence of adherence to treatment and response of cholesterol on mortality in the coronary drug project. N Engl J Med 1980;303:1038-41.

6. Rand CS, Nides M, Cowles MK, et al. Long-term metered-dose inhaler adherence in a clinical trial. The Lung Health Study Research Group. Am J Respir Crit Care Med 1995;152:580-8

7. Epstein LH, Cluss PA. A behavioral medicine perspective on adherence to long-term medical regimens. J Consult Clin Psychol 1982;50:950-71.

8. Shumaker S, Ockene JK, Riekert KA. The handbook of health behavior and change. 3rd ed. New York: Springer, 2008.

9. James PN, Anderson JB, Prior JG, et al. Patterns of drug taking in patients with chronic airflow obstruction. Postgrad Med J 1985;61:7-10

10. Corden ZM, Bosley CM, Rees PJ, et al. Home nebulized therapy for patients with COPD: patient compliance with treatment and its relation to quality of life. Chest 1997;112:1278-82.

11. Nides MA, Tashkin DP, Simmons MS, et al. Improving inhaler adherence in a clinical trial through the use of the nebulizer chronolog. Chest 1993;104:501-7.

\section{Airways disease: just nosing around?}

\section{Glenis K Scadding, Harsha H Kariyawasam}

The airway is a continuous structure extending from the nasal vestibule to the alveoli, with the same pseudostratified ciliated columnar epithelium along much of its length. While an arbitrary line at the level of the vocal cords divides the airway into upper and lower subdivisions-a concept introduced in medical school anatomy tutorials and continued in patient care-airways disease does not conform to such specific anatomical regions. Upper and lower airway disease often coexist, with upper airway involvement often preceding that of the lower airway and even determining severity of disease and quality of life. ${ }^{1}$ This recognition has led to the development of the terms "united airways" and "one airway, one disease".

The nose is an air conditioner-filtering, warming and humidifying over 10000 litres of air daily before it progresses to the lungs. The nasal passages and associated structures bear the brunt

Royal National Throat Nose \& Ear Hospital and Royal Free Hospital NHS Trust, London, UK

Correspondence to: Dr G K Scadding, Royal National Throat Nose \& Ear Hospital, Department of Allergy and Medical Rhinology, 330 Gray's Inn Road, London WC1X 8DA, UK; g.scadding@ucl.ac.uk of environmental contact, being the first site of allergen, microbial and particle deposition. As a consequence, the upper airway is the location of a highly-developed innate and adaptive immune system. Effective mucociliary clearance is vital for respiratory health, as evidenced by the effects of defects such as primary ciliary dyskinesia (PCD) and cystic fibrosis. Sinus disease is almost universal in these patients, and even the subgroup of idiopathic bronchiectasis demonstrates high rates of chronic sinus infection, polyps and inflammation. ${ }^{2}$ Simple measures such as nasal douching can help with symptoms and quality of life. Measurement of nasal nitric oxide is simple and quick, ${ }^{3}$ and very low levels can alert the physician to the possibility of PCD before major lung damage is sustained, thus allowing the benefit of early physiotherapy.

An important lymphoid tissue mass (tonsils and adenoids) collectively termed Waldeyer's ring and prominent in childhood is a unique immunological organ surrounding the upper airway. It supports development and maturation of the immune system. A defective or compromised immune response often manifests initially with recurrent upper airway infection before involving the lungs or other organs. The upper airway epithelium can rapidly generate an array of immunomodulatory cytokines, chemokines and growth factors in response to injury, allergen or pathogen contact that can activate and sustain an airway inflammatory response. The submucosal tissue is rich in antigen presenting cells that can sample the environment, process material and subsequently navigate an immune response via induction of $\mathrm{T}$ cell function and immunoglobulin production. An over-excessive or dysregulated immune response can lead to allergic disease, predominantly Th2-mediated and possibly predisposed to by the presence of thymic stromal lymphopoietin in nasal epithelium. ${ }^{45}$ Alternatively, Th1 mechanisms give rise to serious systemic disorders such as Wegener's granulomatosis in which, again, upper airway manifestations usually appear first. ${ }^{6}$ Early diagnosis and rapid treatment can prevent further organ involvement such as renal disease and improve prognosis. The upper airway is also a site of manifestation of rheumatological disease such as Sjogren's syndrome or disorders of vascular remodelling impairment such as hereditary haemorrhagic telangiectasia. It is therefore evident that a broad spectrum of disease can present at the nasal level before involving the remainder of the airways, allowing a window of opportunity for diagnosis and early intervention.

Rhinitis means inflammation of the nasal lining, but the term is used to define a constellation of symptoms-nasal obstruction or congestion, rhinorrhoea 